\title{
Altered Gene Expression in an Embolic Stroke Model After Thrombolysis With Tissue Plasminogen Activator and Stachybotrys microspora Triprenyl Phenol-7
}

\author{
Terumasa Hashimoto ${ }^{1, *, \dagger}$, Keita Shibata ${ }^{1, \dagger}$, Hisayuki Ohata ${ }^{1}$, Keiji Hasumi ${ }^{2}$, and Kazuo Honda ${ }^{1}$ \\ ${ }^{1}$ Division of Pharmacology, Department of Pharmacology, Toxicology \& Therapeutics, School of Pharmacy, Showa University, \\ 1-5-8 Hatanodai, Shinagawa-ku, Tokyo 142-8555, Japan \\ ${ }^{2}$ Department of Applied Biological Science, Tokyo University of Agriculture and Technology, \\ 3-5-8, Saiwai-cho Fuchu City, Tokyo 183-8509, Japan
}

Received January 26, 2014; Accepted March 24, 2014

\begin{abstract}
The present study compares gene expression and infarct area in a mouse model of embolic stroke after thrombolysis with t-PA and SMTP-7. Embolic occlusion was induced by transfer of acetic acid-induced embolus into the brain. t-PA or SMTP-7 was administered $3 \mathrm{~h}$ after embolization. Changes in gene expression were evaluated using microarray and RT-PCR analysis. To determine the involvement of reactive oxygen species in the response to t-PA, the free radical scavenger edaravone was infused immediately before t-PA administration. The expressions of 459 genes involved in the inflammatory response, cell-to-cell signaling, cell movement, and inflammatory disease were altered by embolic occlusion. Twenty-two of those genes were upregulated after t-PA but not SMTP-7 administration. Differences between the t-PAand SMTP-7-treated groups in the expression of genes including the proinflammatory genes Il6, Stat3, S100a8, and Mmp9 were confirmed with RT-PCR. Edaravone ameliorated the overexpression of these genes. Our data demonstrate differences in gene expression following treatment with SMTP-7 or t-PA that likely explain the difference in therapeutic time windows of the two drugs. ROS are involved in the overexpression of proinflammatory genes. The wide therapeutic time window may be achieved through an anti-oxidative effect and inhibition of proinflammatory gene overexpression.
\end{abstract}

Keywords: SMTP-7, t-PA, ischemia/reperfusion injury, gene expression, inflammation

\section{Introduction}

Thrombolysis therapy using tissue plasminogen activator (t-PA) is highly effective in the early stages of ischemic stroke. Recently, ECASS study revealed that t-PA administration between 3 and $4.5 \mathrm{~h}$ after the onset of symptoms significantly improved clinical outcomes in patients with acute ischemic stroke (1). Thus, now the clinicians in many countries provide treatment within the time window of $4.5 \mathrm{~h}$. However, the risk of hemorrhagic complications beyond $3 \mathrm{~h}$ following

*Corresponding author. t-hashi@pharm.showa-u.ac.jp

"These authors contributed equally to this work.

Published online in J-STAGE on May 1, 2014

doi: 10.1254/jphs.14019FP ischemia significantly increase due to breakdown of blood-brain barrier (2). t-PA can also induce ischemia/ reperfusion injury (3). Therefore, an alternative strategy is needed to extend the therapeutic time window, which would minimize damage from ischemia/reperfusion injury (4). Stachybotrys microspora triprenyl phenol-7 (SMTP-7) has a wide therapeutic time window and results in minimal intracerebral hemorrhage compared with t-PA $(5-7)$. Model mice given SMTP-7 show relatively mild rolling and attachment of leukocytes to the vascular wall following thrombolytic reperfusion (8). These properties of SMTP-7 make it an excellent therapeutic agent for cerebral infarction in this embolic stroke model.

Cerebral ischemia and subsequent reperfusion elicit an inflammatory response, and many genes are differen- 
tially expressed following cerebral ischemia $(9,10)$. Characterization of changes in gene expression that occur during embolic stroke and after therapeutic intervention can illuminate the mechanisms involved in embolic stroke and ischemia/reperfusion injury. Microarray analysis offers an unparalleled way to evaluate changes in the expression of a vast number of genes. We therefore hypothesized that gene expression following SMTP-7induced thrombolysis differs from that following t-PAinduced thrombolysis and that the difference in gene expression may explain the reduction in harmful outcomes.

The aim of the present study was to compare the effect of SMTP-7 and t-PA thrombolysis on gene expression in a mouse model of embolic stroke. We report here the differences in gene expression between SMTP-7- and t-PA-treated mice. Additionally, our investigation suggests that the wide therapeutic time window of SMTP-7 may be attributed to its suppression of proinflammatory gene overexpression following thrombolytic reperfusion.

\section{Materials and Methods}

\section{Animals}

All experiments were conducted in accordance with the regulations of the Committee of Animal Care and Welfare of Showa University.

Male ddY mice weighing 35-45 g (Saitama Jikken, Saitama) were maintained in an air-conditioned room at $20^{\circ} \mathrm{C} \pm 2{ }^{\circ} \mathrm{C}$, with $50 \% \pm 20 \%$ relative humidity and a $12 \mathrm{~h}$ light-dark cycle (lights on 8:00 - 20:00). Animals received a standard laboratory diet and water was provided ad libitum.

\section{Experimental procedures}

The embolic stroke model was induced as previously described $(5,6)$. Animals were anesthetized with $5 \%$ isoflurane (Escain $\AA$; Mylan, Tokyo), and anesthesia was maintained with $1.0 \%-1.5 \%$ isoflurane. Body temperature was maintained by use of a heating lamp throughout surgery and during the recovery period. Under anesthesia, the right common carotid artery (RCCA) was isolated via a midline incision and the surrounding tissue was carefully removed. The RCCA was temporarily clamped using an aneurysm clip and acetic acid was introduced to generate the thrombus. After $10 \mathrm{~min}$, the clip was removed, and the thrombus was transferred to the brain by the bloodstream. Sham-operated animals underwent the same surgical procedure but without application of acetic acid to the RCCA.

For administration of t-PA (10 mg/kg, i.v.) or SMTP-7 $(10 \mathrm{mg} / \mathrm{kg}$, i.v.), $10 \%$ of the dose of either agent was administered as a bolus $3 \mathrm{~h}$ after embolization, followed by infusion of the remaining dose over $30 \mathrm{~min}$. Edaravone
( $3 \mathrm{mg} / \mathrm{kg}$, i.v.) was infused as a bolus immediately before t-PA administration. Control animals received an equivalent volume of saline in place of the thrombolytic agents.

\section{Evaluation of cerebral blood flow (CBF) and infarction area}

Twenty-four hours following embolization, CBF was determined by laser Doppler flowmetry (MoorFLPI; Moor Instruments, Ltd., Millway Axminster, Devon, UK) in accordance with the manufacturer's instructions, and the scanning image was analyzed using the MoorFLPI software (Ver. 2.1). An analysis of the infarction area was conducted as previously described $(5,6)$. The animals were sacrificed with diethylether. Subsequently, brains were removed, sectioned coronally into four 2-mm sections with a brain matrix (RBM 2000C; ASI Instruments, Warren, MI, USA) and incubated with $2 \%$ 2,3,5-triphenyltetrazolium chloride (TTC, Wako Pure Chemical, Osaka) in saline for $30 \mathrm{~min}$ at $37^{\circ} \mathrm{C}$. After TTC staining, brain slices were photographed on the posterior surface of each section and areas of infarction were delineated on the basis of relative lack of staining in the ischemic slice. The infarction areas were measured utilizing Image J software (version 1.31; NIH, Bethesda, MD, USA) and numerically integrated across the thickness of the slice to obtain an estimate of the infarction area and whole brain area respectively. The areas from all slices were summed to calculate the total infarction area and were expressed as percentage of the whole brain area.

\section{RNA extraction and microarray analysis}

Total RNA was extracted from the infarcted area using TRIzol regent (Invitrogen, Carlsbad, CA, USA). Samples were evaluated spectrophotometrically at $260 \mathrm{~nm}$ to determine concentration. Microarray analysis was performed using the one color labeling method with the Quick Amp Labeling Kit (Agilent Technologies, Santa Clara, CA, USA) according to the manufacturer's instructions. Double-stranded cDNA was synthesized with M-MLV reverse transcriptase using a T7 promoter primer. Cy3-labeled cRNA was synthesized using T7 RNA polymerase, purified using the RNeasy Mini Kit (Qiagen, Valencia, CA, USA), and quantified using a spectrophotometer. A $1.65-\mu \mathrm{g}$ sample of Cy3-labeled cRNA was fragmented by incubating it at $60^{\circ} \mathrm{C}$ for 30 min and hybridized to a whole mouse genome DNA microarray ( $4 \times 44 \mathrm{~K}$, Agilent) for $17 \mathrm{~h}$ at $65^{\circ} \mathrm{C}$. After washing, the microarray was scanned with the Agilent microarray scanner. Data were obtained with Agilent Feature Extraction software (version 9.5). Data analysis was performed using Gene Spring GX 10 software (Tomy Digital Biology, Tokyo) and gene functional classification was performed with Ingenuity Pathway 
Analysis (Ingenuity Systems, Redwood City, CA, USA).

Real-time reverse transcription polymerase chain reaction (real-time RT-PCR)

Microarray data were confirmed using real-time RT-PCR. Aliquots $(1 \mu \mathrm{g})$ of total RNA were subjected to a reverse transcription reaction using a SUPERSCRIPT first-strand cDNA synthesis system (Invitrogen). Target cDNA levels were quantified by real-time RT-PCR using the ABI PRISM 7000 Sequence Detector (Applied Biosystems, Foster City, CA, USA) and SYBR GreenER qPCR SuperMix (Invitrogen) in accordance with the manufacturer's instructions. The forward primer for $\beta$ actin was 5 '-CCTTCCTTCTTGGGTATGGAATC-3' and the reverse primer was 5'-TGCTAGGAGCCAGAG CAGTAATC-3'. Other primers were purchased from Qiagen. Gene expression was normalized to $\beta$-actin from the same sample of cDNA.

\section{Statistical analysis}

The affected genes with a greater than threefold change in expression compared with control are listed. Other data are expressed as the mean \pm S.E.M. Student's $t$-test was employed to determine the statistical difference between the sham and the saline group. Multiple comparisons were analyzed by ANOVA followed by the Bonferroni test. $P$-value less than 0.05 was considered significant.

\section{Results}

Thrombolytic reperfusion with SMTP-7, but not $t-P A$, reduced infarct area in a model of embolic stroke

The effects of thrombolytic agents on an acetic acidinduced embolic stroke model were evaluated (Fig. 1). Cerebral infarction was observed $24 \mathrm{~h}$ after embolization (infarct area $10.6 \% \pm 5.31 \%$ in the saline group, compared with $2.99 \% \pm 0.27 \%$ in the sham group). A slight increase in infarct area was observed in mice that received t-PA $(15.3 \% \pm 4.53 \%)$ compared with those that received saline, although this did not reach statistical significance. However, the infarct area in the SMTP-7treated group was significantly smaller than that in the t-PA-treated and saline groups and not different from that in the sham group. By contrast, $\mathrm{CBF}$ at $24 \mathrm{~h}$ after embolization recovered to about $70 \%$ of the initial value after administration of either agent; no significant difference in $\mathrm{CBF}$ was observed between the t-PA and SMTP-7 groups.

Thrombolytic reperfusion with $t-P A$, but not SMTP-7, induced overexpression of pro-inflammatory genes

Microarray analysis of cerebral tissue was carried out to investigate the influence of thrombolytic reperfusion on gene expression. The expression of a large number of genes in the cerebral tissue changed more than threefold in the model group compared with that of the sham group (upregulated: 1321 genes, downregulated: 769 genes). Notably, expression of 459 genes involved in the inflammatory response, cell-to-cell signaling and interaction, cell movement, and inflammatory disease was greatly altered (Fig. 2), with 22 of these genes being upregulated more than threefold after t-PA administration compared with the saline administration (Table 1). The same effect was not seen after administration of SMTP-7 (Fig. 3).

\section{Verification of proinflammatory gene expression using real-time $R T-P C R$}

To confirm the changes in gene expression identified by microarray, four genes (Il6, Stat3, S100a8, and Mmp9) were chosen for analysis with real-time RT-PCR (Fig. 4). A significant overexpression of Il6, Stat3, and S100a8 was observed after ischemia. This overexpression was enhanced by t-PA administration. However, SMTP-7 administration did not induce overexpression of these genes, and Il6 and S100a8 expression was lower in mice that received SMTP-7 than in those that received saline. In the case of $M m p 9$, overexpression was also observed in mice that received t-PA, although statistical significance was not observed between the saline and sham group. In contrast, gene expression in the SMTP7-treated group was smaller than that in the t-PA-treated group and not different from that in the sham group. Thus, RT-PCR analysis confirmed the direction of transcriptional regulation observed with microarray analysis, although the technique showed smaller fold changes than did microarray analysis.

Edaravone reduced cerebral infarction and prevented proinflammatory gene overexpression

To investigate whether the upregulation of Il6, Stat3, S100a8, and $\mathrm{Mmp} 9$ expression after ischemia/reperfusion is related to the production of reactive oxygen species (ROS), we examined the cerebral infarct area and expression of the four genes after combined administration of edaravone and t-PA. Infarct area and proinflammatory gene expression in the saline group were significantly higher in sham group. A combination of t-PA and edaravone greatly reduced infarct area and a significant difference was observed compared with the t-PA-treated group (Fig. 5A). Similarly, RT-PCR analysis indicated that the upregulation of these genes observed after t-PA administration was ameliorated when the drug was combined with edaravone (Fig. 5B). 




B)
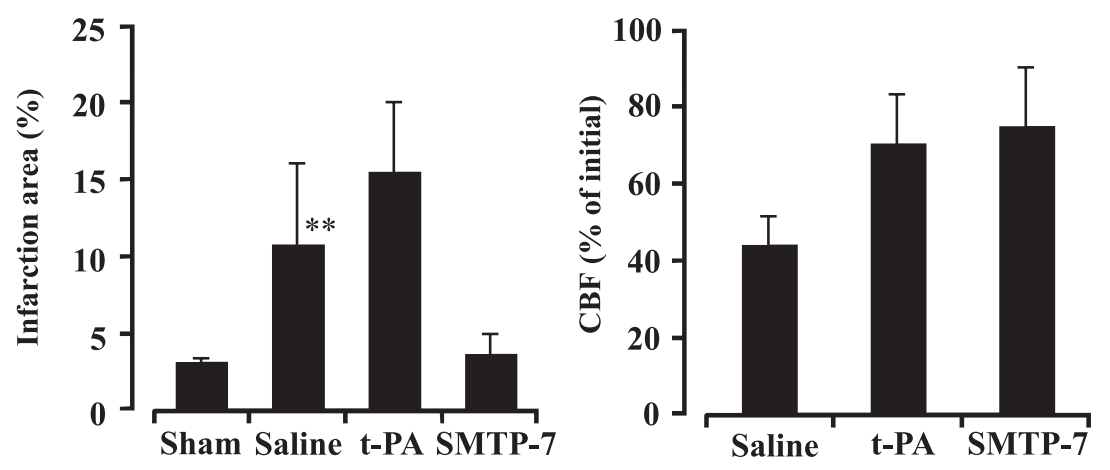

Fig. 1. Cerebral infarction after administration of t-PA, SMTP-7, or saline in a mouse model of embolic stroke. A) Coronal sections (2-mm thickness) showing 2,3,5-triphenyltetrazolium chloride-stained cerebral tissue, $24 \mathrm{~h}$ after embolization. Arrows indicate infarction area. B) Mean \pm S.E.M. cerebral infarction area $(\mathrm{n}=5-6$ per group) and CBF ( $\mathrm{n}=3$ per group). $* * P<0.01$, compared with the sham group.



Fig. 2. Gene expression profile in post-ischemic brain. The 10 canonical pathways with the greatest fold change from the sham group are shown, identified by Ingenuity Pathway Analysis in the ischemic core.

\section{Discussion}

In the present study, we compared the effects of SMTP-7- and t-PA-induced thrombolysis on gene
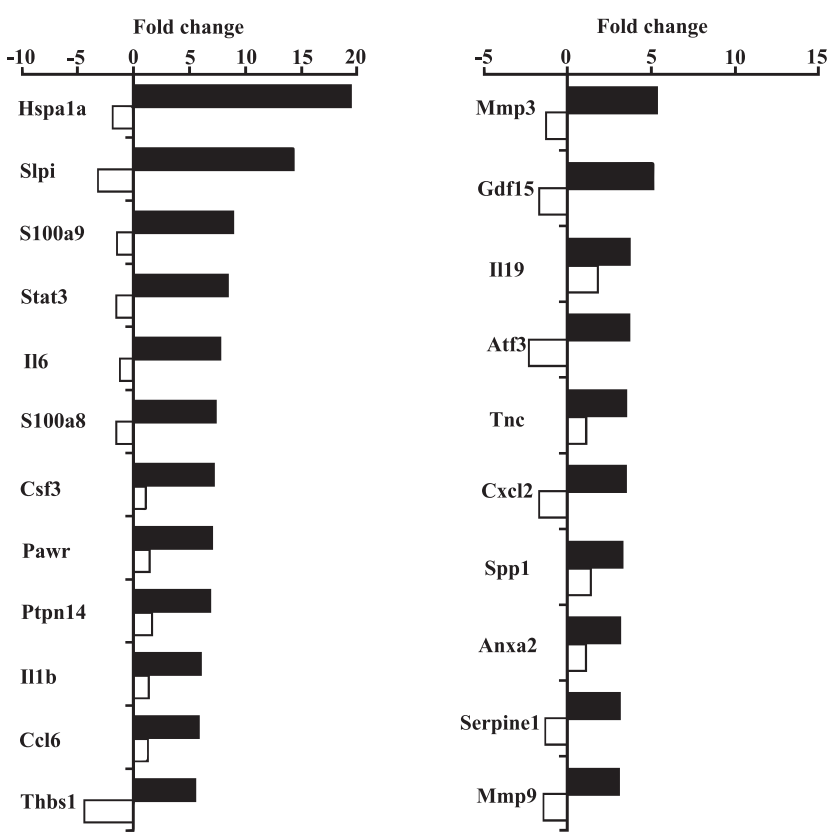

Fig. 3. Gene expression after reperfusion with t-PA and SMTP-7. Each value is expressed as fold change compared with saline administration. Closed column: t-PA, Open column: SMTP-7.

expression in a mouse model of embolic stroke, to follow up our previous observations that SMTP-7 has a wide therapeutic time window and minimizes hemorrhage compared with t-PA $(5-7)$. Here, a slight increase in infarct area was observed in t-PA-treated model mice compared with the saline group, but not in SMTP-7treated model mice. CBF recovered to around $70 \%$ of 
Table 1. Microarray analysis. Genes with more than threefold upregulation after administration of t-PA compared with saline

\begin{tabular}{|c|c|c|}
\hline Gene symbol & Gene name & Fold \\
\hline Hspa1a & Heat shock $70 \mathrm{kDa}$ protein $1 \mathrm{~A}$ & 19.3 \\
\hline Slpi & Secretory leukocyte peptidase inhibitor & 14.2 \\
\hline S100a9 & S100 calcium binding protein A9 & 8.81 \\
\hline Stat3 & Signal transducer and activator of transcription 3 & 8.34 \\
\hline Il6 & Interleukin 6 & 7.67 \\
\hline $\mathrm{S} 100 \mathrm{a} 8$ & S100 calcium binding protein $\mathrm{A} 8$ & 7.26 \\
\hline Csf3 & Colony stimulating factor 3 & 7.09 \\
\hline Pawr & PRKC, apoptosis, WT1, regulator & 6.94 \\
\hline Ptpn14 & Protein tyrosine phosphatase, non-receptor type 14 & 6.76 \\
\hline Il1B & Interleukin 1 , beta & 5.93 \\
\hline Ccl6 & Chemokine (C-C motif) ligand 6 & 5.73 \\
\hline Thbs 1 & Thrombospondin 1 & 5.43 \\
\hline Mmp3 & Matrix metallopeptidase 3 & 5.28 \\
\hline Gdf15 & Growth differentiation factor 15 & 5.05 \\
\hline I119 & Interleukin 19 & 3.66 \\
\hline Atf3 & Activating transcription factor 3 & 3,63 \\
\hline Tnc & Tenascin $\mathrm{C}$ & 3.45 \\
\hline $\mathrm{Cxcl} 2$ & Chemokine (C-X-C motif) ligand 2 & 3.43 \\
\hline Spp1 & Secreted phosphoprotein 1 & 3.22 \\
\hline Anxa2 & Annexin A2 & 3.09 \\
\hline Serpine1 & Serpin peptidase inhibitor, clade E (nexin, plasminogen activator inhibitor type 1 ), member 1 & 3.06 \\
\hline Mmp9 & Matrix metallopeptidase 9 & 3.01 \\
\hline
\end{tabular}
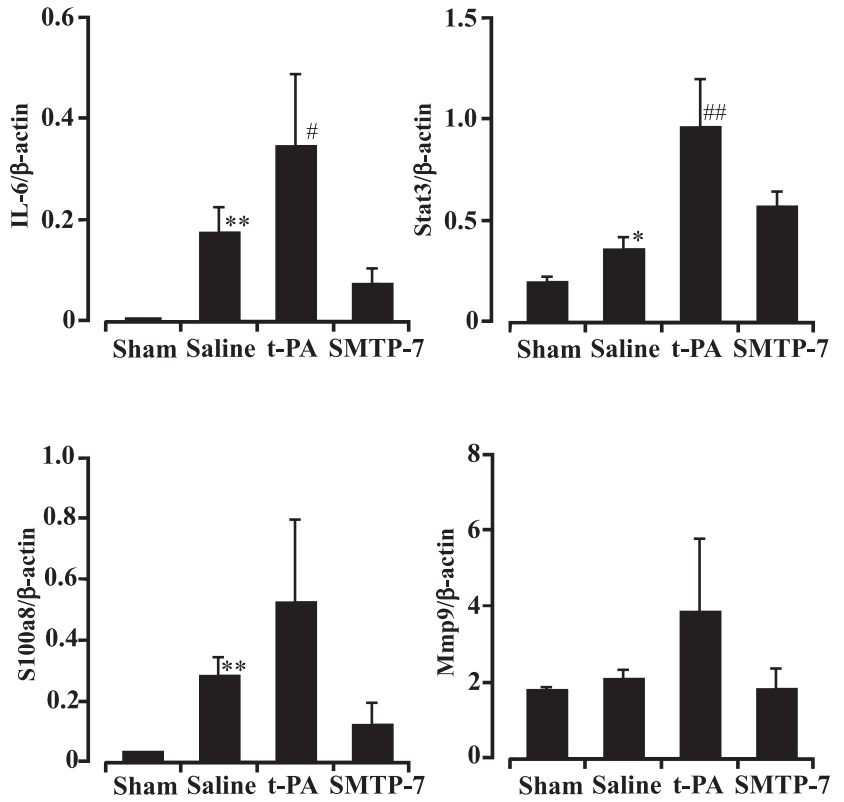

Fig. 4. Effects of t-PA and SMTP-7 on mRNA expression of IL6, Stat3, S100a8, and Mmp 9 in the ischemic core after embolic infarction (mean \pm S.E.M. of 6 experiments). $* P<0.05$, $* * P<0.01$, compared with the sham group; ${ }^{\#} P<0.05,{ }^{\#} P<0.01$, compared with the saline group. the pre-ischemic value after administration of either drug; therefore, administration of t-PA, but not SMTP-7, induced ischemia/reperfusion injury.

Using microarray analysis, we have identified 22 genes associated with ischemia/reperfusion injury after t-PA administration. Although it is widely known that various inflammatory mediators such as cytokines and chemokines are related to ischemia/reperfusion injury (11), enhancement of Hspla, Csf, Thbs 1, and Serpine1 expression was also observed in the present study. Upregulation of $70-\mathrm{kDa}$ heat shock protein ( Hsp 70) mRNA reflects the severity of ischemic stress (12) and represents a protective mechanism during ischemia (13). Expression of Thbs1, a major angiostatic factor, also occurs after ischemia (14). Moreover, treatment with granulocyte colony stimulating factor or plasminogen activator inhibitor-1 reduces infarct volume after ischemia/reperfusion $(15,16)$. From these reports, we hypothesized that enhancement of the expression of these genes results in negative feedback, which contributes to ischemia/reperfusion injury. In SMTP-7-treated mice, proinflammatory gene expression was not enhanced. Therefore, SMTP-7 does not facilitate ischemia/reperfusion injury or enhance the expression of the genes in the hypothesized negative feedback loop.

We paid particular attention to the expression of Il6, Stat3, S100a8, and Mmp9. Signal transducer and 
B)
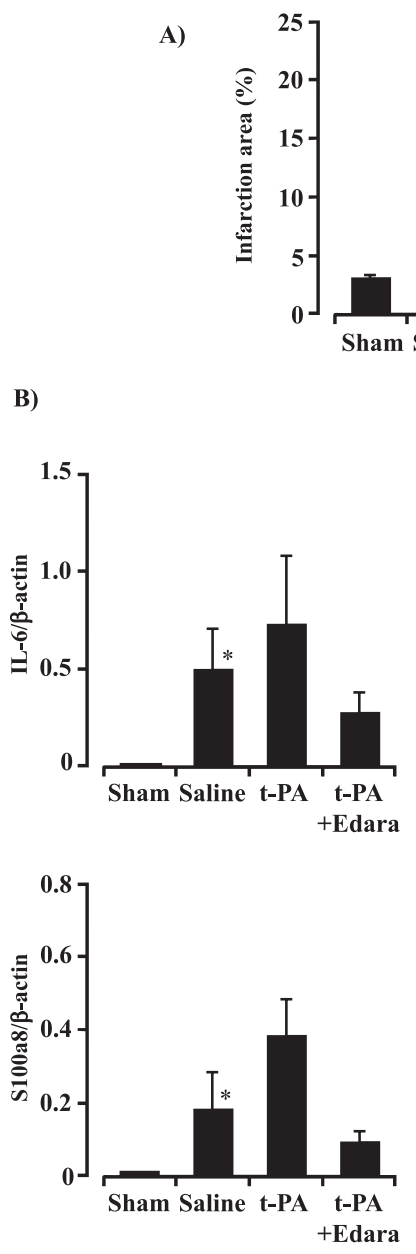

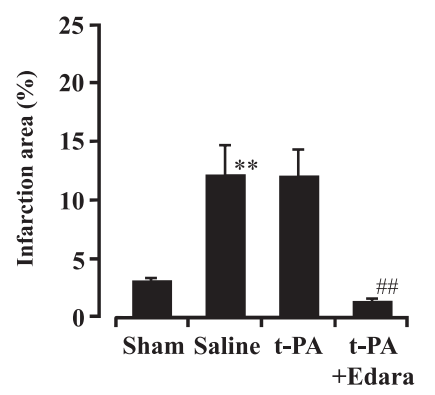

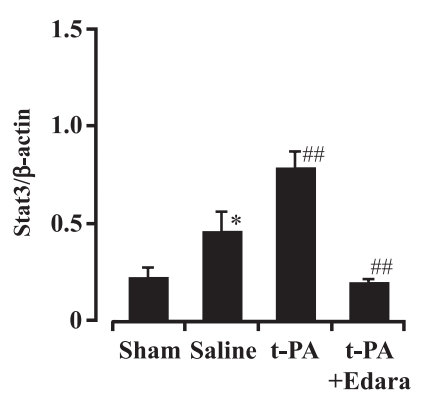

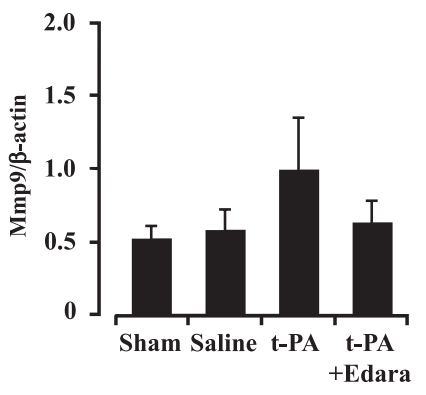

Fig. 5. Effect of edaravone on cerebral infarction and mRNA expression in embolic infarction model. A) Cerebral infarction. B) mRNA expression. Mean \pm S.E.M. of 6 experiments. $* P<0.05$, ${ }^{*} P<0.01$, compared with the sham group; ${ }^{\#} P<0.01$, compared with the saline group.

activator of transcription (STAT) is induced by various stimuli, and activated STAT translocates into the nucleus and activates the transcription of its target genes (17). STAT expression is increased by ischemia (18), STAT3 activation is related to ROS production, and inhibition of STAT3 activation leads to a decrease in infarct volume induced by focal cerebral ischemia/reperfusion injury (19). Excessive production of ROS also induces upregulation of $I l 6$, and antioxidants attenuate this overexpression (20). Furthermore, STAT3 is a downstream target of IL-6 (21). The response to IL-6 binding leads to the homodimerization and translocation of STAT3 to the nucleus. Toll-like receptor-4 (TLR-4) is involved in ischemia/reperfusion injury (22) and activates the nuclear factor $\kappa-\mathrm{B}(\mathrm{NF}-\kappa \mathrm{B})$ signaling pathway, which is linked to the transcription of proinflammatory genes such as Mmp9. The active form of Mmp9 appears after ischemia/reperfusion (23) and this is attributed to the oxidative stress-associated breakdown of the blood-brain barrier and ischemia/reperfusion-induced hemorrhage via oxidative stress (24). Additionally, S100A8 and S100A9, members of the S100 calciumbinding protein family, are characterized as endogenous activators of TLR-4 (25); overexpression of these genes was found after ischemia/reperfusion (26). From these reports, we selected the genes Il6, Stat3, S100a8, and Mmp9 and verified their expression with real-time RT-PCR.

Consistent with the microarray, RT-PCR analysis indicated an increase in Il6, Stat3, S100a8, and Mmp9 expression in the model mice after t-PA administration and an inhibitory effect of SMTP-7 on this overexpression. Mmp9 is proteolytic enzyme and cleavage is needed to change it to the active form. Protein level or enzymatic activity of Mmp9 was not examined in the present study. However, a recent study showed that SMTP-7 suppressed activation of Mmp9 and superoxide production (27). From these results and the previous repot, we theorized that the wide therapeutic time window of SMTP-7 could be attributed to the inhibitory effect on the expression of these proinflammatory genes after ischemia/reperfusion. In addition, this is one reason why SMTP-7 appears to cause less hemorrhagic conversion (6). Some of the transcription factors known to be involved in gene expression are regulated by ROS $(28-30)$. Therefore, the action of SMTP-7 may be attributable to its antioxidative action because superoxide production after transient focal cerebral ischemia was suppressed in SMTP-7-treated rats (27). NF- $\kappa \mathrm{B}$ is one of the best characterized transcription factors activated by ROS (31). However, in the present study, this gene expression was not affected by t-PA administration (below threefold change), although Stat3 expression was significant greater than that in the saline-treated group. Although it is difficult to identify which transcription factor pathway contributes most to ischemia/reperfusion injury, we propose that the STAT3 pathway is involved at least in part, because of the upregulation of Stat3 after t-PA administration.

Next, we examined the effects of edaravone on the upregulation of gene expression induced by t-PA to verify whether ROS production was involved in the overexpression of proinflammatory genes after ischemia/ reperfusion. Combined administration of edaravone with t-PA not only reduced the infarct area but also ameliorated the overexpression of proinflammatory genes. It is reported that additive administration of edaravone ameliorates the oxidative damage induced by t-PA (32). From the present results and previous reports, we conclude that ROS is an important mediator 
of the response to ischemia/reperfusion, and scavenging of ROS is correlated with the expansion of the therapeutic time window in thrombolysis therapy using SMTP-7 via suppression of proinflammatory gene expression.

In summary, the expression of 22 genes associated with ischemia/reperfusion injury is enhanced after thrombolysis with t-PA but not with SMTP-7. This difference in gene expression may explain the wide therapeutic time window of SMTP-7; moreover, we propose that the mechanism behind the expanded therapeutic time window of this agent involves the suppression of the overexpression of transcription factor genes such as Stat3.

\section{Acknowledgments}

This work was supported by a Private University High Technology Research Center Project matching fund subsidy from MEXT (Ministry of Education, Culture, Sports, Science and Technology)-supported program for the strategic research foundation at private university. The funding bodies had no role in the study design, data collection or analysis, writing of the report, or the decision to submit the article for publication. The authors would like to thank TMS Co., Ltd. (Tokyo) for generously supplying SMTP-7.

\section{Conflicts of Interest}

The authors declare that there are no conflicts of interest.

\section{References}

1 Hacke W, Kaste M, Bluhmki E, Brozman M, Dávalos A, Guidetti $\mathrm{D}$, et al; ECASS Investigators. Thrombolysis with alteplase 3 to 4.5 hours after acute ischemic stroke. N Engl J Med. 2008; 359:1317-1329.

2 Su EJ, Fredriksson L, Geyer M, Folestad E, Cale J, Andrae J, et al. Activation of PDGF-CC by tissue plasminogen activator impairs blood-brain barrier integrity during ischemic stroke. Nat Med. 2008;14:731-737.

3 Yepes M, Roussel BD, Ali C, Vivien D. Tissue-type plasminogen activator in ischemic brain: more than a thrombolytic. Trends Neurosci. 2009;32:48-55.

4 Jaffer H, Morris VB, Stewart D, Labhasetwar V. Advances in stroke therapy. Drug Deliv Transl Res. 2011;1:409-419.

5 Hashimoto T, Shibata K, Nobe K, Hasumi K, Honda K. A novel embolic model of cerebral infarction and evaluation of Stachybotrys microspora triprenyl phenol-7 (SMTP-7), a novel fungal triprenyl phenol metabolite. J Pharmacol Sci. 2010;114: 41-49.

6 Shibata K, Hashimoto T, Nobe K, Hasumi K, Honda K. A novel finding of low-molecular-weight compound, SMTP-7, having thrombolytic and anti-inflammatory effects in cerebral infarction of mice. Naunyn-Schmied Arch Pharmacol. 2010;382:245-252.

7 Sawada H, Nishimura N, Suzuki E, Zhuang J, Hasegawa K, Takamatsu H, et al. SMTP-7, a novel small-molecule thrombolytic for ischemic stroke: a study in rodents and primates. $\mathrm{J}$ Cereb Blood Flow Metab. 2014;34:235-241.

8 Miyazaki T, Kinura Y, Ohata H, Hashimoto T, Shibata K, Hasumi
$\mathrm{K}$, et al. Distinct effect of tissue-type plasminogen activator and SMTP-7 on cerebrovascular inflammation following thrombolytic reperfusion. Stroke. 2011;42:1097-1104.

9 Jin K, Mao XO, Eshoo MW, Nagayama T, Minami M, Simon RP, et al. Microarray analysis of hippocampal gene expression in global cerebral ischemia. Ann Neurol. 2001;50:93-103.

10 Kim YD, Sohn NW, Kang C, Soh Y. DNA array reveals altered gene expression in response to focal cerebral ischemia. Brain Res Bull. 2002;58:491-498.

11 Wang Q, Tang XN, Yenari MA. The inflammatory response in stroke. J Neuroimmunol. 2007;184:53-68.

12 Hara T, Mies G, Hata R, Hossmann KA. Gene expressions after thrombolytic treatment of middle cerebral artery clot embolism in mice. Stroke. 2001;32:1912-1919.

13 Weinstein PR, Hong S, Sharp FR. Molecular identification of the ischemic penumbra. Stroke. 2004;35:2666-2670.

14 Lin TN, Kim GM, Chen JJ, Cheung WM, He YY, Hsu CY. Differential regulation of thrombospondin-1 and thrombospondin-2 after focal cerebral ischemia/reperfusion. Stroke. 2003;34:177186.

15 Nagai N, Suzuki Y, Van Hoef B, Lijnen HR, Collen D. Effects of plasminogen activator inhibitor-1 on ischemic brain injury in permanent and thrombotic middle cerebral artery occlusion models in mice. J Thromb Haemost. 2005;3:1379-1384.

16 Komine-Kobayashi M, Zhang N, Liu M, Tanaka R, Hara H, Osaka A, et al. Neuroprotective effect of recombinant human granulocyte colony-stimulating factor in transient focal ischemia of mice. J Cereb Blood Flow Metab. 2006;26:402-413.

17 Hebenstret D, Horejs-Hoeck J, Duschl A. JAK/STAT-dependent gene regulation by cytokines. Drug News Perspect. 2005;18: 243-249.

18 Sun SL, Li TJ, Yang PY, Qiu Y, Rui YC. Modulation of signal transducers and activators of transcription (STAT) factor pathways during focal cerebral ischemia: a gene expression array study in rat hippocampus after middle cerebral artery occlusion. Clin Exp Pharmacol Physiol. 2007;34:1097-1101.

19 Lei C, Deng J, Wang B, Cheng D, Yang Q, Dong H, et al. Reactive oxygen species scavenger inhibits STAT3 activation after transient focal cerebral ischemia-reperfusion injury in rats. Anesth Analg. 2011;113:153-159.

20 Bowler RP, Sheng H, Enghild JJ, Pearlstein RD, Warner DS, Crapo JD. A catalytic antioxidant (AEOL 10150) attenuates expression of inflammatory genes in stroke. Free Radic Biol Med. 2002;15:1141-1152.

21 Levy DE, Lee CK. What does Stat3 do? J Clin Invest. 2002; 109:1143-1148.

22 Caso JR, Pradillo JM, Hurtado O, Lorenzo P, Moro MA, Lizasoain I. Toll-like receptor 4 is involved in brain damage and inflammation after experimental stroke. Circulation. 2007; 115:1599-1608.

23 Fujimura M, Gasche Y, Morita-Fujimura Y, Massengale J, Kawase M, Chan PH. Early appearance of activated matrix metalloproteinase-9 and blood-brain barrier disruption in mice after focal cerebral ischemia and reperfusion. Brain Res. 1999; 842:92-100.

24 Gasche Y, Copin JC, Sugawara T, Fujimura M, Chan PH. Matrix metalloproteinase inhibition prevents oxidative stress-associated blood-brain barrier disruption after transient focal cerebral ischemia. J Cereb Blood Flow Metab. 2001;21:1393-1400.

25 Vogl T, Tenbrock K, Ludwig S, Leukert N, Ehrhardt C, van 
Zoelen MA, et al. Mrp8 and Mrp14 are endogenous activators of Toll-like receptor 4, promoting lethal, endotoxin-induced shock. Nat Med. 2007;13:1042-1049.

26 Ziegler G, Prinz V, Albrecht MW, Harhausen D, Khojasteh U, Nacken W, et al. Mrp-8 and -14 mediate CNS injury in focal cerebral ischemia. Biochim Biophys Acta. 2009;1792:1198-1204.

27 Akamatsu Y, Saito A, Fujimura M, Shimizu H, Mekawy M, Hasumi K, et al. Stachybotrys microspora triprenyl phenol-7, a novel fibrinolytic agent, suppresses superoxide production, matrix metalloproteinase- 9 expression, and thereby attenuates ischemia/reperfusion injury in rat brain. Neurosci Lett. 2011; 503:110-114.

28 Irani K, Xia Y, Zweier JL, Sollott SJ, Der CJ, Fearon ER, et al. Mitogenic signaling mediated by oxidants in Ras-transformed fibroblasts. Science. 1997;275:1649-1652.
29 Schreck R, Albermann K, Baeuerle PA. Nuclear factor kappa B: an oxidative stress-responsive transcription factor of eukaryotic cells. Free Radic Res Commun. 1992;17:221-237.

30 Simon AR, Rai U, Fanburg BL, Cochran BH. Activation of the JAK-STAT pathway by reactive oxygen species. Am J Physiol. 1998;275:C1640-C1652.

31 Clemens JA. Cerebral ischemia: gene activation, neuronal injury, and the protective role of antioxidants. Free Radic Biol Med. 2000;28:1526-1531.

32 Lukic-Panin V, Deguchi K, Yamashita T, Shang J, Zhang X, Tian $\mathrm{F}$, et al. Free radical scavenger edaravone administration protects against tissue plasminogen activator induced oxidative stress and blood brain barrier damage. Curr Neurovasc Res. 2010; 7:319-329. 